\title{
Add-on effect of clarithromycin to oral steroids as post- operative therapy for chronic rhinosinusitis with nasal polyps: a randomised controlled trial*
}

\author{
Chien-Fu Lin', Mao-Che Wang ${ }^{2,3}$, Arlene T. Merton ${ }^{4}$, Nien-Hsuan Ho², \\ Pao-Shu Wu $\mathrm{W}^{5}$, Angela Ting-Wei Hsu' ${ }^{6}$, Ying-Piao Wang ${ }^{1,7,8}$ \\ ' Department of Otolaryngology-Head and Neck Surgery, Mackay Memorial Hospital, Taipei, Taiwan \\ 2 Department of Otolaryngology-Head and Neck Surgery, Taipei Veterans General Hospital, Taipei, Taiwan \\ ${ }^{3}$ Institute of Public Health and Community Medicine Research Centre, National Yang-Ming University, Taipei, Taiwan \\ ${ }^{4}$ Department of Otolaryngology, Vicente Sotto Memorial Medical Centre, Cebu, Philippines \\ ${ }^{5}$ Department of Pathology, Mackay Memorial Hospital, Taipei, Taiwan \\ ${ }^{6}$ Department of Biostatistics and Epidemiology, The Johns Hopkins Bloomberg School of Public Health, Baltimore, MD, USA \\ School of Medicine, Mackay Medical College, New Taipei City, Taiwan \\ ${ }^{8}$ Department of Audiology and Speech Language Pathology, Mackay Medical College, New Taipei City, Taiwan
}

Rhinology 58: 6, $550-558,2020$

https://doi.org/10.4193/Rhin 19.325

*Received for publication:

September 19, 2019

Accepted: March 8, 2020

\begin{abstract}
Background: Evidence is lacking regarding the efficacy of macrolides and oral corticosteroids in chronic rhinosinusitis with nasal polyps (CRSwNP) after endoscopic sinus surgery (ESS). Therefore, we examined the benefits of adding clarithromycin to oral prednisolone as post-ESS medical therapy in patients with CRSwNP.
\end{abstract}

Methods: In this randomised, double-blind, placebo-controlled trial, patients were enrolled and allocated to three study groups receiving different post-ESS medical therapies: group A (placebo for 14 weeks), group B (oral prednisolone [15 mg twice daily] for 2 weeks, followed by placebo for 12 weeks), and group C (oral prednisolone [15 mg twice daily] for 2 weeks, followed by clarithromycin [500 mg daily] for 12 weeks). All enrolled patients received the perioperative care following a routine protocol, which included oral amoxicillin/clavulanate, and intranasal corticosteroid spray. The baseline and post-operative visual analogue scale (VAS) scores, Sino-nasal Outcome Test (SNOT-22) scores, and Lund-Kennedy endoscopy scores (LKES) were determined as the primary outcomes.

Results: One hundred twenty-six patients who received ESS for bilateral CRSwNP were randomised into group A $(n=43), B(n=42)$, or $C(n=41)$. Compared to groups A and B, group C showed greater VAS and SNOT-22 score improvement at 12 weeks after ESS. Group $C$ showed significantly better LKES than did groups $A$ and $B$ at 8, 12, and 24 weeks after ESS. On stratifying the LKES results according to the presence/absence of tissue eosinophilia, greater add-on effects of clarithromycin were observed in the patient subgroup without tissue eosinophilia.

Conclusions: Adding low-dose clarithromycin to oral corticosteroids as post-ESS therapy was well tolerated and showed beneficial subjective and objective outcomes in patients with CRSwNP, especially those without tissue eosinophilia.

Key words: clarithromycin, nasal polyps, paranasal sinus disease, post-operative care, sinusitis

\section{Introduction}

Chronic rhinosinusitis with nasal polyps (CRSwNP) accounts for $20-30 \%$ of chronic rhinosinusitis (CRS), and affects $1.1-2.7 \%$ of the general population worldwide ${ }^{(1-4)}$. Endoscopic sinus surgery (ESS) is a safe and effective surgical intervention for medically recalcitrant CRSWNP(1). Nevertheless, post-ESS recurrence accounts 
for a big share of CRS-related disease burden and health care $\operatorname{cost}^{(5,6)}$. Current guidelines recommend aggressive post-operative medical therapy with office-based debridement, saline irrigation, and intranasal corticosteroid spray (INCS) to minimise recurrence $\mathrm{e}^{(1,7)}$. The pathophysiology of nasal polyp recurrence involves both infectious and inflammatory processes of the sinonasal mucosa. Consequently, attention has been focused on post-ESS corticosteroids and macrolides, which have wellknown anti-inflammatory and immunomodulatory effects ${ }^{(8)}$. Corticosteroids can effectively control eosinophilic inflammation by inhibiting eosinophil recruitment to the inflammation site, suppressing interleukin- 5 release, and inducing eosinophil apoptosis $^{(9)}$. Reportedly, $61.3 \%$ of patients with CRSwNP were treated with oral corticosteroids (OCSs) in the Global Allergy and Asthma European Network rhinosinusitis cohort in 2019(10). Both the European Position Paper on Rhinosinusitis and Nasal Polyps (EPOS) 2012 guidelines and Poetker et al.'s systematic review strongly suggested OCS for the perioperative medical treatment for CRSwNP ${ }^{(1,11)}$. Two randomised controlled trials (RCTs) by Wright et al. and Shen et al. demonstrated significantly better endoscopy scores in patients with CRSwNP receiving post-ESS OCS than in those receiving a placebo ${ }^{(7,12)}$. Nonetheless, other evidence showed no significant benefits of peri-operative OCS in patients with $\mathrm{CRS}^{(13,14)}$.

Macrolides, beside their anti-microbial effects, have immunomodulatory effects associated with the suppression of neutrophil activity and down-regulation of interleukin-8, nuclear factor-кB, and tumour necrosis factor- $\mathrm{a}^{(15,16)}$. Recent systematic reviews have established the benefits of macrolides in the treatment of CRS without nasal polyps (CRSsNP), but controversy exists about whether they have similar effects in CRSwNP(17-23). To date, only four RCTs addressing the post-operative effects of long-term, low-dose macrolides (LDMs) have been published in the English literature, but these yielded conflicting conclusions ${ }^{(24-27)}$.

The use of corticosteroids and macrolides is well established in the treatment of lower airway diseases, such as asthma and chronic obstructive pulmonary disease (COPD) $)^{(9,15,28-33)}$. OCSs have been the backbone of treatment in acute asthma exacerbations, wherein eosinophilic inflammation and Thelper 2 cytokines play important roles ${ }^{(9)}$. In contrast, macrolides are effective in preventing acute exacerbations of COPD ${ }^{(28,29,33)}$ which mainly involve the activation of neutrophils, macrophages, and Thelper 1 cells $^{(33)}$. Recent review articles suggested combination therapy with OCSs and LDMs for managing refractory COPD coexistent with asthma (previously referred to as asthma-COPD overlap syndrome), which involves both eosinophilic and neutrophilic airway inflammation ${ }^{(30,34)}$.

Similarly, CRSwNP is considered a heterogeneous disease with mixed eosinophilic and neutrophilic inflammation. Since RCTs and review articles yielded inconclusive results regarding the

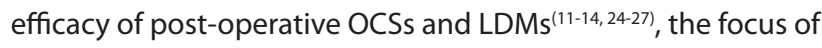

recent research has shifted to identifying subgroups of patients with CRSwNP that can benefit from post-operative OCSs and LDMs. Two endotypes of CRSwNP, namely eosinophilic CRSwNP (eCRSwNP) and non-eosinophilic CRSwNP (neCRSwNP), are expected to have differential responsiveness to corticosteroids and macrolides, but this hypothesis is as yet unconfirmed ${ }^{(35-37)}$. Although combination therapy with OCS and LDM is a potential solution to control both eosinophilic and neutrophilic inflammation, opinion is still divided about its efficacy after ESS in patients with CRSwNP(26, 27).

Therefore, we conducted a three-arm, double-blind, placebocontrolled, randomised clinical trial focusing on the efficacy of combination therapy with oral prednisolone and clarithromycin immediately after ESS in patients with CRSwNP, along with subgroup analyses to examine the responsiveness of OCSs and LDMs in different endotypes of CRSwNP.

\section{Material and methods}

\section{Study design}

This single-centre, single-surgeon, prospective three-arm, double-blind, randomised, placebo-controlled trial was conducted at the Department of Otorhinolaryngology-Head and Neck Surgery, Mackay Memorial Hospital, Taipei, Taiwan. The study protocol was approved by the Institutional Review Board of Mackay Memorial Hospital, and all enrolled patients signed informed consent forms.

\section{Patient selection}

Patients with bilateral CRSwNP who had unsuccessful maximal medical treatment and subsequently received primary or revised bilateral ESS at Mackay Memorial Hospital were enrolled. The diagnosis of CRS was based on the definition established by the EPOS 2012 guidelines ${ }^{(1)}$. Maximal medical treatment comprised empirical oral antibiotics with amoxicillin/clavulanate, INCS, and saline nasal irrigation for at least 4 weeks with or without oral antihistamines.

The exclusion criteria were age younger than 18 years, fungal sinusitis, odontogenic sinusitis, pregnancy, lactation, sinonasal tumours, underlying liver dysfunction, and concomitant medication with drugs interacting with prednisolone or clarithromycin. Patients who had received systemic corticosteroids and/or macrolides for other medical purposes were also excluded.

\section{Sample size calculation}

On the basis of a pilot study on 25 patients with bilateral CRSwNP and using visual analogue scale (VAS) scores at 1 month after ESS as the primary outcome, we calculated a required sample size of 102 , with an effect size $F=0.312$, a value $=0.05$, and $80 \%$ power. Considering follow-up loss, a total sample size of 126 was planned consequently. 


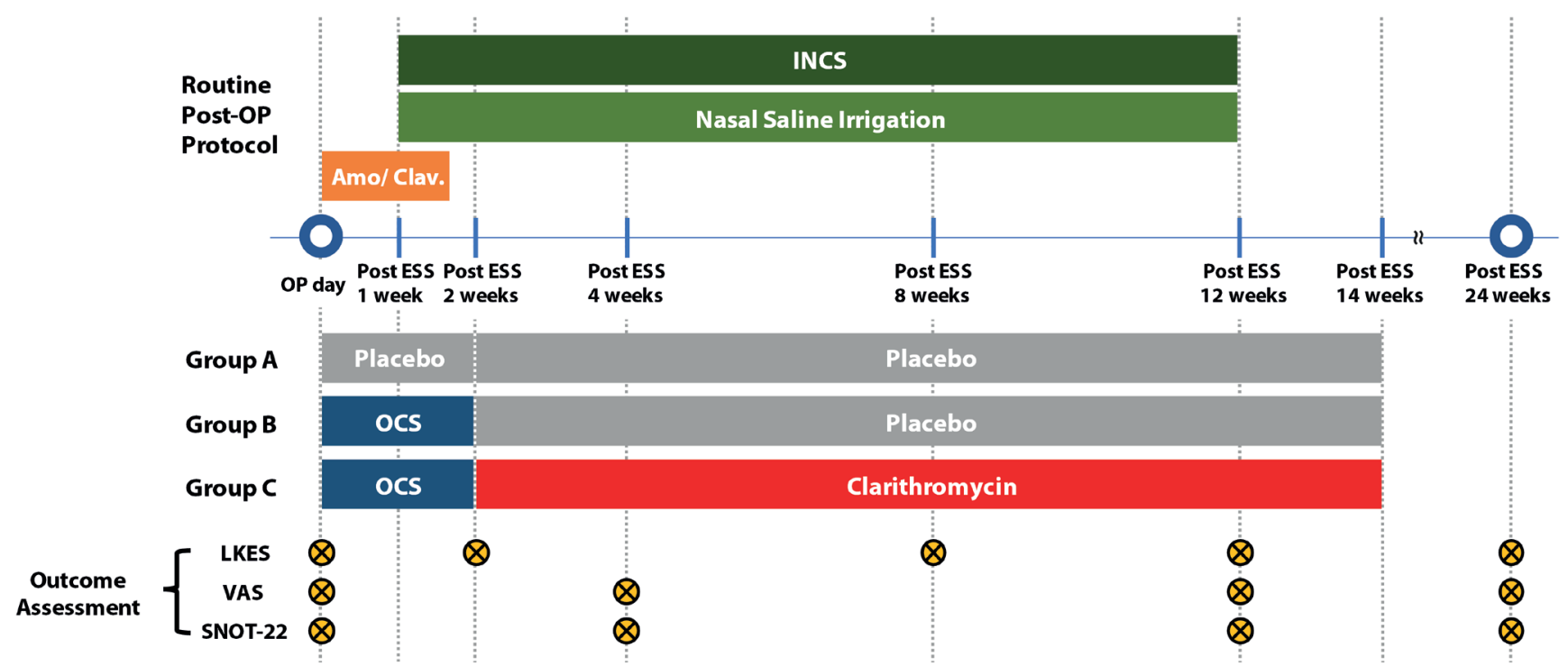

Figure 1. Study timeline. Illustration of routine post-operative protocol, duration of study medications, and the schedule of follow-up visits. (INCS, intranasal corticosteroids spray; Amo/Clav., amoxicillin/clavulanate; ESS, endoscopic sinus surgery; OCS, oral corticosteroid; LKES, Lund-Kennedy endoscopy score; VAS, visual analogue scale; SNOT-22, Sino-Nasal Outcome Test).

\section{Surgical technique and peri-operative protocol}

All patients underwent paranasal sinus computed tomography (CT) as pre-operative assessment, and the CT images were graded using Lund-Mackay scores (LMS). All patients underwent bilateral ESS performed by the same surgeon (Dr. Ying-Piao Wang) using the Messerklinger technique as described by Stammberger and Kennedy ${ }^{(38,39)}$. The extent of surgery was individually designed for each patient according to the disease extent on paranasal sinus $\mathrm{CT}$. The mucosa was preserved as much as possible, and healthy sinuses were not dissected unless needed for better exposure of diseased ones. Specimens of the patients' sinonasal mucosa were sent to the Pathology Department of Mackay Memorial Hospital, where they were stained with haematoxylin and eosin and inspected under a microscope. Eosinophil count per high-power field (HPF, 400x) was calculated by the same pathologist (Dr. Pao-Shu Wu). Tissue eosinophilia was defined as more than 10 eosinophils per HPF as described in previous studies ${ }^{(40-43)}$.

A routine protocol was applied for the post-operative management of all patients. Oral amoxicillin/clavulanate ( $1 \mathrm{~g}$ twice daily) was prescribed after ESS for 10 days. Patients were instructed to start nasal saline irrigation twice daily, 1 week after ESS. INCS with mometasone furoate ( $100 \mu \mathrm{g}$ once daily) was started 1 week after ESS and was continued until 12 weeks after ESS. Intranasal debridement was performed 1, 2, and 4 weeks after ESS in an office-based manner to remove crust, discharge, and degraded packing materials (Figure 1).

\section{Study medication}

All patients were randomly allocated to one of three groups: group A (placebo for 14 weeks), group B (oral prednisolone [15 mg twice daily] for 2 weeks, followed by placebo for 12 weeks), and group C (oral prednisolone [15 mg twice daily] for 2 weeks, followed by clarithromycin [500 mg daily] for 12 weeks) (Figure 1). A randomisation list was generated using a computer program at the Department of Medical Research, Mackay Memorial Hospital, with 1:1:1 simple randomisation; the list was confidentially stored at the institution. A copy of the list was sent to the pharmacy of Mackay Memorial Hospital, where the study medications (i.e. clarithromycin and prednisolone tablets) were packed into capsules. Identical empty capsules served as placebo. Identical dosage instructions were provided to all three groups of patients.

\section{Subjective outcome assessment}

For subjective outcome assessment, the VAS and Chinese version of Sino-nasal Outcome Test (SNOT-22) scores were evaluated at baseline and 4, 12, and 24 weeks after ESS. On the VAS, nine major parameters were graded: nasal obstruction, rhinorrhoea, post-nasal drip, anosmia, headache, facial pain, facial fullness, cacosmia, and cough (individual scores, 0-10; total score range, $0-90$ ). SNOT-22 contains 22 items (individual scores, $0-5$; total score range, $0-110)$ reflecting sinus-specific quality of life (QoL) $(44,45)$. On both the VAS and SNOT-22, higher scores represent more severe symptoms and worse QoL.

\section{Objective outcome assessment}

We used the Lund-Kennedy endoscopy score (LKES) to assess objective outcomes at baseline and 2, 8, 12, and 24 weeks after ESS. The LKES was based on endoscopic findings made by two 


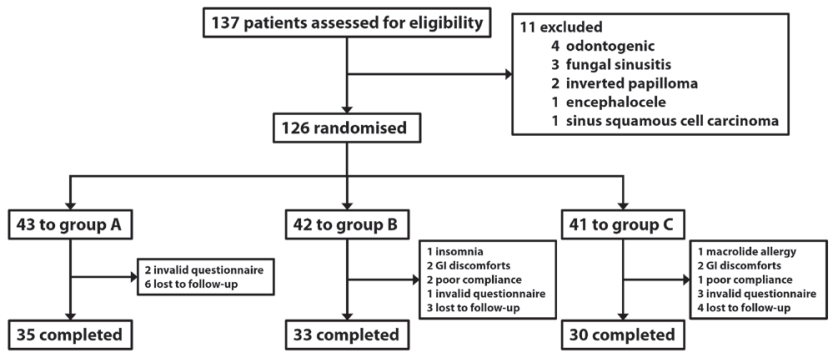

Figure 2. Study flowchart.

individual otolaryngologists who were blinded to the treatment. Re-evaluation was performed in cases of discrepancies. The LKES includes five different items: polyps, oedema, discharge, scarring, and crusting (individual scores, $0-2$ ), and the average scores of both sides are calculated (range, $0-10$ ), with higher scores representing more unfavourable endoscopic findings ${ }^{(46)}$.

\section{Statistical analyses}

Outcomes were evaluated regardless of study medication discontinuation, as this study followed the intention-to-treat principle. Descriptive data were presented as percentages and means \pm standard deviations. One-way analysis of variance was used to compare continuous variables. Bonferroni test was used for post-hoc analyses. In subgroup analyses, Kruskal-Wallis $\mathrm{H}$ test was used to compare primary outcomes, and Dunn's test was used for post-hoc analyses, because of the relatively small sample size. Pearson's Chi-square test was used for comparing categorical variables. Statistical analyses were performed using IBM SPSS Statistics for Windows, Version 21.0 (IBM Corp., Armonk, NY, USA). A p-value less than 0.05 was considered statistically significant.

\section{Results}

Between January 2015 and December 2018, 137 patients were assessed for eligibility and 126 were included and randomised to group A (placebo, $n=43$ ), group B (oral prednisolone, $n=42$ ) or group $C$ (oral prednisolone + clarithromycin, $\mathrm{n}=41$ ). Among them, $77.8 \%(98 / 126)$ completed the trial at 24 weeks after ESS, with $81.4 \%$ (35/43), 78.6\% (33/42), and 73.2\% (30/41) completing the protocol in groups $A, B$, and $C$, respectively (Figure 2). The demographic characteristics of the study groups are listed in Table 1. No significant difference was found among the three arms in terms of age, sex, smoking, previous operation, asthma, aspirin allergy, and tissue eosinophilia. The baseline polyp grading, LMS, LKES, VAS scores, and SNOT-22 scores were all balanced among the groups.

\section{VAS scores}

The baseline VAS scores were $37.98 \pm 16.30$ in group $A$, $34.08 \pm 14.85$ in group $B$, and $38.70 \pm 15.95$ in group $C$, with no significant intergroup difference $(p=0.356)$ (Table 1). At the 12-

Table 1. Baseline characteristics of the three study groups.

\begin{tabular}{|c|c|c|c|c|}
\hline & Placebo (A) & OCS + Placebo (B) & OCS + LDM (C) & p-value \\
\hline Number & 43 & 42 & 41 & \\
\hline Sex & & & & 0.282 \\
\hline Male & $32(74.4 \%)$ & $33(78.6 \%)$ & $26(63.4 \%)$ & \\
\hline Female & $11(25.6 \%)$ & $9(21.4 \%)$ & $15(36.6 \%)$ & \\
\hline Age & $46.72( \pm 16.49)$ & $44.88( \pm 15.03)$ & $48.24( \pm 12.78)$ & 0.589 \\
\hline Smoking & $10(23.3 \%)$ & $9(21.4 \%)$ & $9(22.0 \%)$ & 0.978 \\
\hline Previous operation & 19 (44.2\%) & $10(23.8 \%)$ & 15 (36.6\%) & 0.138 \\
\hline Asthma & $6(14.0 \%)$ & $5(11.9 \%)$ & $8(19.5 \%)$ & 0.606 \\
\hline Aspirin allergy & $3(6.3 \%)$ & $4(9.5 \%)$ & $6(14.6 \%)$ & 0.503 \\
\hline Tissue eosinophilia (>10/HPF) & $27(62.8 \%)$ & $29(69.0 \%)$ & $25(61.0 \%)$ & 0.722 \\
\hline Serum eosinophil count $/ \mu \mathrm{L}$ & $269.63( \pm 311.84)$ & $281.32( \pm 323.25)$ & $287.72( \pm 271.50)$ & 0.923 \\
\hline Serum eosinophil \% & $3.39( \pm 3.17)$ & $3.92( \pm 4.63)$ & $3.95( \pm 3.55)$ & 0.978 \\
\hline Serum IgE level (IU/ml) & $141.03( \pm 225.32)$ & $138.43( \pm 168.15)$ & $109.99( \pm 175.67)$ & 0.515 \\
\hline Polyp grading & $2.01( \pm 1.03)$ & $1.89( \pm 0.99)$ & $2.02( \pm 1.11)$ & 0.570 \\
\hline Lund-Mackay score & $14.07( \pm 5.92)$ & $13.98( \pm 4.81)$ & $15.49( \pm 5.08)$ & 0.350 \\
\hline LKES & $3.51( \pm 0.70)$ & $3.30( \pm 0.76)$ & $3.27( \pm 0.65)$ & 0.238 \\
\hline VAS score & $37.98( \pm 16.30)$ & $34.08( \pm 14.85)$ & $38.70( \pm 15.95)$ & 0.356 \\
\hline SNOT-22 score & $49.28( \pm 22.04)$ & $45.17( \pm 19.66)$ & $48.88( \pm 18.64)$ & 0.589 \\
\hline
\end{tabular}

(OCS, oral corticosteroid; LDM, low-dose macrolide; HPF, high power field; IgE, immunoglobulin E; LKES, Lund-Kennedy endoscopy scores; VAS, visual analogue scale; SNOT-22, Sino-Nasal Outcome Test). 


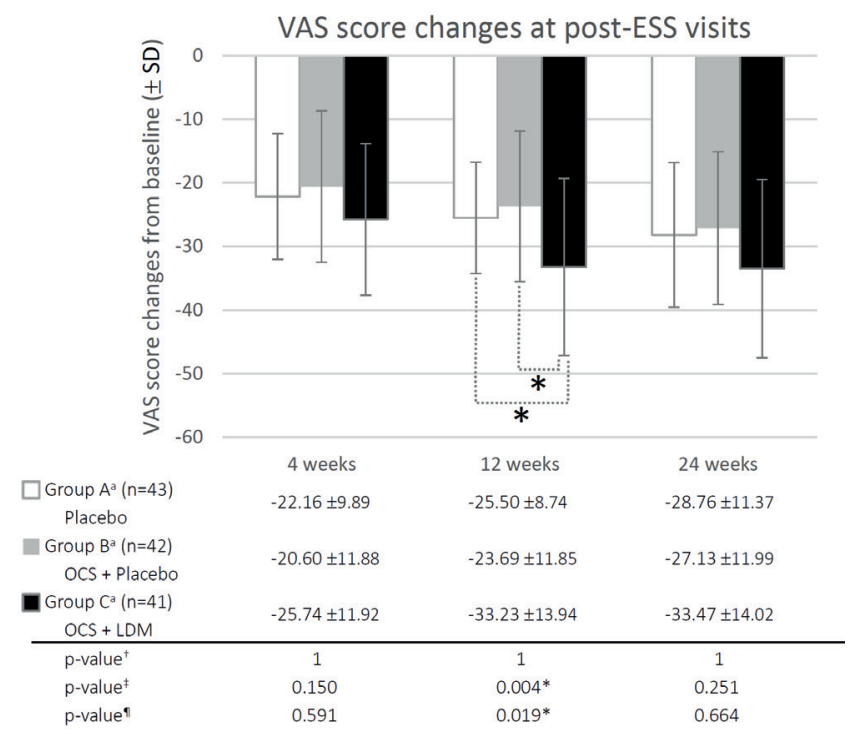

Figure 3. Visual analogue scale (VAS) score changes from baseline of the three study groups at visits 4, 12, and 24 weeks after endoscopic sinus surgery (ESS). (SD, standard deviation; OCS, oral corticosteroid; LDM, lose-dose macrolide). a Baseline VAS scores of group A, B, and C were $37.98 \pm 16.30,34.08 \pm 14.85$, and $38.70 \pm 15.95$, respectively. ${ }^{+}$Comparison between group $\mathrm{A}$ (placebo) and group $\mathrm{B}$ (OCS + placebo). ${ }^{\ddagger}$ Comparison between group $B(O C S$ + placebo) and group C (OCS + LDM).

${ }^{9}$ Comparison between group A (placebo) and group C (OCS + LDM).

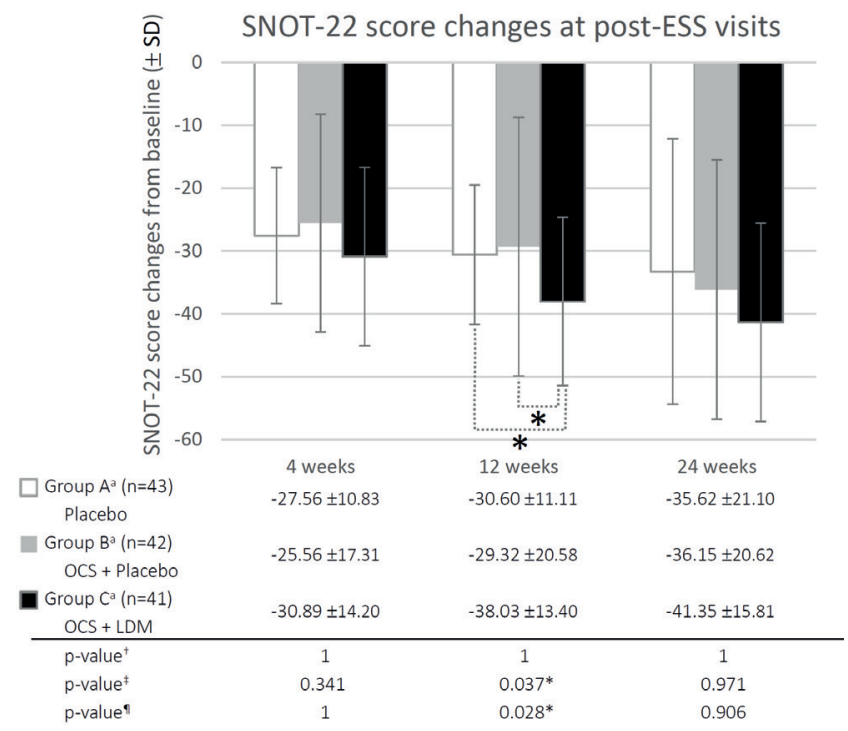

Figure 4. Sino-Nasal Outcome Test (SNOT-22) score changes from baseline of the three study groups at visits 4, 12, and 24 weeks after endoscopic sinus surgery (ESS). (SD, standard deviation; OCS, oral corticosteroid; LDM, lose-dose macrolide). a Baseline SNOT-22 scores of group $A, B$, and $C$ were $49.28 \pm 22.04,45.17 \pm 19.66$, and 48.88 \pm 18.64 , respectively. ${ }^{+}$Comparison between group $A$ (placebo) and group $B$ (OCS + placebo). ${ }^{\ddagger}$ Comparison between group $\mathrm{B}$ (OCS + placebo) and group $\mathrm{C}$ (OCS + LDM). "Comparison between group A (placebo) and group C $(\mathrm{OCS}+\mathrm{LDM})$. week visit after ESS, the average VAS score changes from baseline were significantly greater in group $C(-33.23 \pm 13.94)$ than in groups $A(-25.50 \pm 8.74 ; p=0.019)$ and $B(-23.69 \pm 11.85 ; p=0.004)$, while no significant difference was found between groups $A$ and B. No significant difference in VAS score change was observed among the groups at the 4- and 24-week visits after ESS (Figure 3).

\section{SNOT-22 scores}

The baseline SNOT-22 scores were $49.28 \pm 22.04$ in group A, $45.17 \pm 19.66$ in group $B$, and $48.88 \pm 18.64$ in group $C$, with no significant intergroup difference $(p=0.589)$ (Table 1). At the 12week visit after ESS, the average SNOT-22 score changes from baseline were significantly greater in group C (-38.03 \pm 13.40$)$ than in groups $A(-30.60 \pm 11.11 ; p=0.028)$ and $B(-29.32 \pm 20.58$; $p=0.037$ ), while no significant difference was found between groups A and B. No significant difference in VAS score change was observed among the groups at the 4- and 24-week visits after ESS (Figure 4).

\section{Endoscopic evaluation (LKES)}

The baseline endoscopy scores were $3.51 \pm 0.70$ in group $A$, $3.30 \pm 0.76$ in group $B$, and $3.27 \pm 0.65$ in group $C(p=0.238)$

(Table 1). No significant intergroup difference was observed at
2 weeks after ESS. At 8 weeks, however, group C demonstrated a significantly better mean LKES $(1.91 \pm 1.35)$ than did groups $A$ $(2.80 \pm 1.24 ; p=0.016)$ and $B(2.57 \pm 1.36 ; p=0.042)$, and the significance was sustained to 24 weeks after ESS, with a mean LKES of $0.94 \pm 0.97$ in group $C$ versus $2.70 \pm 1.10$ in group $A(p<0.001)$ and $1.55 \pm 1.26$ in group $B(p=0.036)$ (Figure 5$)$. Notably, at 24 weeks after ESS, group B $(1.55 \pm 1.26)$ also showed significantly better LKES than did group $A(2.70 \pm 1.10 ; p=0.001)$.

\section{Subgroup analyses}

According to the presence or absence of tissue eosinophilia ( $>10$ eosinophils per HPF), the patients were stratified into the eCRSwNP and neCRSwNP subgroups. The eCRSwNP subgroup included 27 patients in group A, 29 in group B, and 25 in group C. The LKES at 24 weeks after ESS in groups $B(1.57 \pm 1.30)$ and $C(1.40 \pm 1.02)$ were significantly lower than that in group $A$ $(2.92 \pm 1.11 ; p=0.003$ and 0.001 , respectively), while no significance was found at 2,8, and 12 weeks after ESS. No statistically significant difference was found between groups $B$ and $C$ at any visit (Figure 6).

The neCRSwNP subgroup included 16 patients in group $A, 13$ in group $B$, and 16 in group C. Group C demonstrated significantly lower LKES than did group A at 8, 12, and 24 weeks after ESS (all $p<0.001)$ as well as group B at 8 and 24 weeks after ESS ( $p=0.047$ 


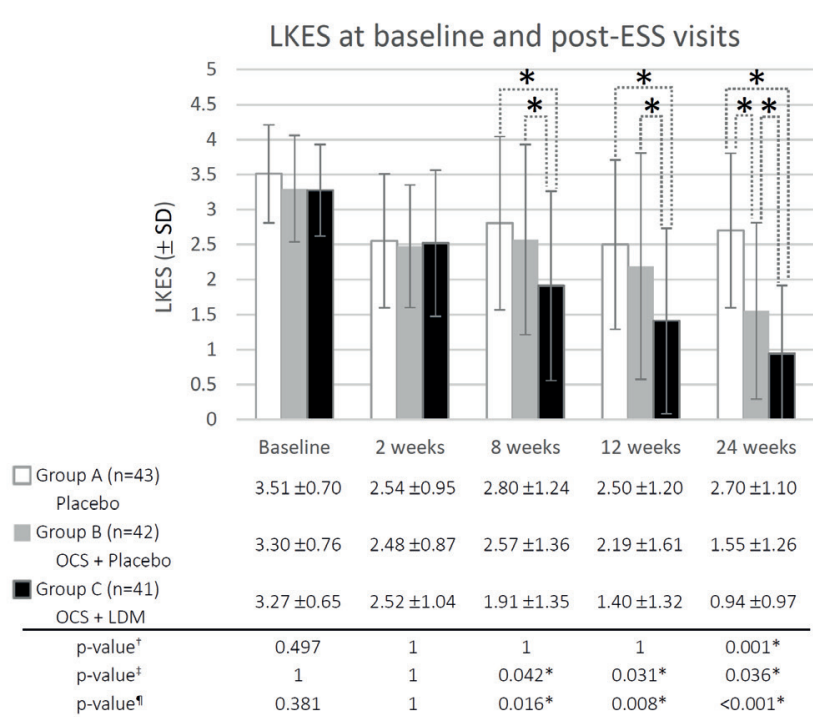

Figure 5. Lund-Kennedy endoscopy scores (LKES) of the three study groups at baseline and 2, 8, 12, 24 weeks after endoscopic sinus surgery (ESS). (SD, standard deviation; OCS, oral corticosteroid; LDM, lose-dose macrolide). +Comparison between group A (placebo) and group B (OCS + placebo), ‡Comparison between group B (OCS + placebo) and group $\mathrm{C}(\mathrm{OCS}+\mathrm{LDM})$, ๆComparison between group $\mathrm{A}$ (placebo) and group $\mathrm{C}$ (OCS + LDM).

and 0.021 , respectively). Compared to group A, group B showed lower LKES at 12 weeks after ESS ( $p=0.007$ ) (Figure 6).

None of the patients with eCRSwNP or neCRSwNP showed statistically significant differences between groups in the VAS and SNOT-22 scores (data not shown).

\section{Discussion}

ESS has become a mainstay in the treatment of CRSwNP that is unresponsive to maximal medical treatment. Nevertheless, a nasal polyp recurrence rate as high as $35 \%$ at 6 months after ESS was reported in a recent multicentre cohort study ${ }^{(47)}$. Aggressive post-operative medical therapy is mandatory to minimise recurrence and optimise post-ESS QoL ${ }^{(48)}$. The EPOS 2012 guidelines mentioned both short-term OCSs (level la, grade A) and long-term LDMs (level Ib, grade C) as part of the post-operative management for CRSwNP(1).

Given the potential risk of side effects, OCSs have still been reserved as a rescue therapy in cases of relapsed symptoms during post-operative follow-up. The efficacy of routine use of short-term OCSs in the immediate post-ESS period is still under debate. RCTs by Wright et al. and Shen et al. demonstrated significantly better endoscopy scores but did not show any benefit in subjective parameters among patients with CRSwNP receiving post-operative short-term OCSs rather than placebo ${ }^{(7,12)}$.

Rudmik et al. and Dautremont et al. do not recommend the administration of peri-operative OCSs to patients with CRSwNP owing to the lack of benefits and risk of adverse effects ${ }^{(13,14)}$. In a retrospective study, Tajudeen et al. reported tissue eosinophil aggregates to be associated with increased OCS requirement in patients with CRS after ESS ${ }^{(37)}$, which implied that eCRSwNP was a corticosteroid-dependent subgroup. Thus far, strong evidence comparing the differential responsiveness to post-operative short-term OCSs in different endotypes of CRS (i.e. eCRSwNP and neCRSwNP) is still lacking.

Four RCTs regarding the efficacy of long-term LDMs in the immediate post-operative period in patients with CRSwNP have been published in the English literature, but their conclusions have been inconsistent. A double-blind, placebo-controlled RCT performed by Amali et al. showed no significant SNOT-22 score

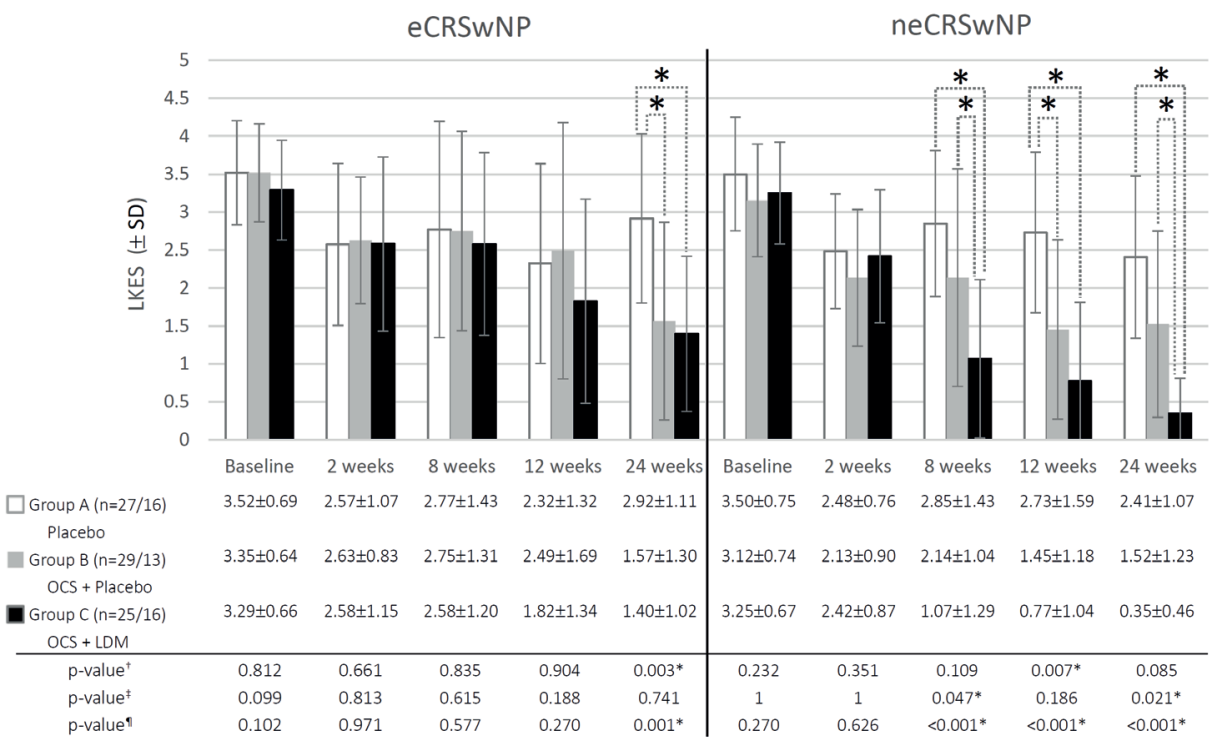

Figure 6. Lund-Kennedy endoscopy scores (LKES) results stratified by different endotypes of chronic rhinosinusitis with nasal polyps (CRSwNP) according to presence/ absence of tissue eosinophilia. (eCRSwNP, eosinophilic CRSwNP; neCRSWNP, noneosinophilic CRSwNP; SD, standard deviation; OCS, oral corticosteroid; LDM, losedose macrolide). + Comparison between group A (placebo) and group B (OCS + placebo). ¥Comparison between group $\mathrm{B}$ (OCS + placebo) and group C (OCS + LDM). ๆComparison between group $A$ (placebo) and group C (OCS + LDM). 
difference between azithromycin ( $250 \mathrm{mg} /$ day for 12 weeks) and placebo among patients with CRSWNP and CRSsNP after ESS ${ }^{(24)}$. Another double-blind, placebo-controlled RCT conducted by Haxel et al., which compared erythromycin $(250 \mathrm{mg} /$ day for 12 weeks) versus placebo among patients with CRSwNP and CRSsNP after ESS, failed to demonstrate significant differences in SNOT-20 and VAS scores but showed significantly better endoscopy scores in the erythromycin group at 12 weeks after ESS ${ }^{(25)}$. Varvyanskaya and Lopatin performed a RCT on patients with CRSwNP after ESS, and showed that patients receiving clarithromycin ( $250 \mathrm{mg} /$ day) for 24 weeks had significantly better SNOT-20, VAS, and endoscopy scores than did those not receiving clarithromycin ${ }^{(26)}$. A more recent $\mathrm{RCT}$ conducted by Zeng et al. did not show significant differences in VAS or endoscopy scores between post-operative clarithromycin $(250 \mathrm{mg}$ / day for 3 months) and INCS treatment for patients with either CRSsNP or CRSwNP ${ }^{(27)}$. Wallwork et al. suggested the possibility of a subgroup of macrolide responders among patients with $\mathrm{CRS}$, and their study found that macrolides were only effective in patients with low serum immunoglobin E levels $(<200 \mathrm{IU} / \mathrm{mL})^{(36)}$. A recent case-control study by Oakley et al. revealed that macrolide response correlated with the lack of tissue eosinophilia( ${ }^{(35)}$. Nevertheless, more evidence is required to confirm the differential macrolide responsiveness in different CRSwNP endotypes. In this study, we demonstrated that the mean VAS and SNOT-22 scores in group C (OCS + LDM) were significantly better than those in groups $A$ (placebo) and B (OCS + placebo) at 12 weeks after ESS, which indicated that long-term, low-dose clarithromycin improved sinus-related symptoms and QoL after ESS. The effects were not sustained at 24 weeks after ESS ( 12 weeks after clarithromycin discontinuation) (Figures 3 and 4). Seresirikachorn et al.'s meta-analysis favoured the 24 -week duration of LDM therapy over shorter durations, but this result should be interpreted with caution because only one included study used 24-week LDM therapy ${ }^{(19)}$. Whether LDM therapy of longer duration (e.g. 24 weeks) can bring extra benefits deserves further investigation. As for post-operative short-term OCS treatment, no significant effects in subjective outcomes could be demonstrated in this study, as no significant difference was observed in the mean VAS and SNOT-22 scores between groups A and B at any visit (Figures 3 and 4).

The add-on effects of long-term, low-dose clarithromycin on post-operative endoscopy scores were also demonstrated by our results, as group C (OCS + LDM) yielded significantly lower mean LKES than did groups A (placebo) and B (OCS + placebo). In group $C$, more healthy-looking mucosa was observed not only during the period of clarithromycin therapy (i.e. from 2 weeks to 14 weeks after ESS) but also at 24 weeks after ESS (12 weeks after clarithromycin discontinuation) (Figure 5). Subgroup analyses demonstrated that the add-on effect of clarithromycin was significant in the neCRSwNP subgroup, while no extra benefits of adding clarithromycin was observed in the eCRSwNP subgroup (Figure 6). This finding echoes those of previous studies that suggested neCRSwNP to be a macrolide-responsive subgroup ${ }^{(26,35)}$.

Our study also found that short-term, moderate-dose OCS seemed to have a delayed effect on the LKES at 24 weeks after ESS (Figure 5). Notably, in subgroup analyses, similar delayed effects of OCS on the LKES were observed in the eCRSwNP subgroup at 24 weeks and in the neCRSwNP subgroup at 12 weeks (Figure 6). Thus, we can deduce that short-term OCS may help prevent post-ESS recurrence of both eCRSwNP and neCRSwNP. However, it was difficult to explain why OCSs, which were administered for only 2 weeks after ESS, started to show their effects at 12-24 weeks after ESS but not earlier. The mechanism underlying this delayed effect warrants further investigation. Of the 83 patients who received oral prednisolone and/or clarithromycin in this study, only 2 (4.8\%) in group B and 2 (4.9\%) in group $C$ had mild gastrointestinal discomfort, and $1(2.4 \%)$ in group B had insomnia. One patient (2.4\%) in group $C$ reported an allergic skin reaction after clarithromycin administration. None of the patients in group A reported adverse effects after taking the study medications. Moreover, no patients reported serious adverse effects.

We proposed the idea of individualised post-ESS medical therapy based on histopathologic endotypes. In this study, the pathology reports were readily available within 1 week after ESS; thus, we were able to stratify every patient into either the eCRSwNP or neCRSwNP subgroup at the first post-ESS visit. Considering the exclusive benefit of post-ESS LDMs in patients with neCRSwNP, as demonstrated in our study, we recommend post-ESS, long-term LDM therapy to be prescribed only to these patients, as indicated by histopathologic findings (i.e. tissue eosinophil count $\leq 10 / \mathrm{HPF}$ ). In countries or hospitals where pathologic report generation takes longer, predicting CRSwNP endotypes by using peripheral blood eosinophil counts serves as a reasonable alternative, as it has been corroborated by previous studies ${ }^{(49,50)}$. Our study also showed that even under the circumstances of no endotype stratification, a combination of short-term, moderate-dose OCS and long-term LDM can be a safe and effective choice for post-ESS medical therapy. Despite these strengths, our study has several limitations. First, although we had estimated the sample size according to the results of a pilot study, we still failed to demonstrate any significance in subjective outcomes in subgroup analyses. Second, the relatively short 24-week observation period might be insufficient to assess the long-term effects of LDM. Third, to avoid cumulative side effects and simplify the placebo protocol, we designed LDMs to be administered in a sequential manner, rather than a concurrent manner, following OCS therapy. The safety and efficacy of concurrent therapy with OCSs and LDMs have to be confirmed by future studies. Fourth, owing to 
the lack of a macrolide-only group in our study, differentiating between the efficacy of LDMs per se and the synergistic effects between OCS and LDM was difficult.

Nevertheless, to our knowledge, this was the first randomised, double-blind, placebo-controlled trial focusing on the add-on effects of post-operative long-term LDMs to short-term OCS with stratification based on different endotypes.

\section{Conclusion}

Our study showed that adding on post-operative long-term (12-week), low-dose (500 mg daily) clarithromycin significantly improved the objective outcomes at 8, 12, and 24 weeks after ESS as well as the subjective outcomes at 12 weeks after ESS, whereas short-term (2-week), moderate-dose (15 mg twice daily) oral prednisolone only yielded more healthy-looking mucosa under endoscopy at 24 weeks after ESS, but provided no benefits to the subjective outcomes. In subgroup analyses, the add-on effects of clarithromycin were more significant in the subgroup of patients without tissue eosinophilia. The results of the current study suggest that adding on low-dose clarithromycin to oral prednisolone should be considered as part of the post-operative medical therapy for patients with CRSwNP, especially for the subgroup of patients without tissue eosinophilia.

\section{Acknowledgements}

We thank Dr. Kuang-Hsuan Shen, Dr. Yu-Hsuan Wang and their colleagues (Department of Otolaryngology-Head and Neck Surgery, Mackay Memorial Hospital) for experience sharing and assistance in data collection.

\section{Authorship contribution}

CF Lin did endoscopic examinations, assessed the endoscopic findings, analysed the data and prepared manuscript; MC Wang participated in data discussion; Arlene Merton assessed the endoscopic findings; $\mathrm{NH}$ Ho participated in data discussion; PS Wu performed histopathologic examinations; Angela TW Hsu assisted data recording and analysed the data; YP Wang designed the study, performed surgeries and prepared manuscript.

\section{Conflict of interest}

There is no conflict of interest

\section{References}

1. Fokkens WJ, Lund VJ, Mullol J, Bachert C, Alobid I, Baroody F, et al. EPOS 2012 European position paper on rhinosinusitis and nasal polyps 2012. A summary for otorhinolaryngologists. Rhinology. 2012;50(1):1-12.

2. Johansson L, Akerlund A, Holmberg K, Melen I, Bende M. Prevalence of nasa polyps in adults: the Skovde populationbased study. Ann Otol Rhinol Laryngol. 2003;112(7):625-9.

3. Shi JB, Fu QL, Zhang $H$, Cheng L, Wang YJ, Zhu DD, et al. Epidemiology of chronic rhinosinusitis: results from a cross-sectional survey in seven Chinese cities. Allergy. 2015;70(5):533-9.

4. Zhang Y, Gevaert E, Lou H, Wang X, Zhang L, Bachert $C$, et al. Chronic rhinosinusitis in Asia. J Allergy Clin Immunol. 2017;140(5):1230-9.

5. Bhattacharyya N, Orlandi RR, Grebner J, Martinson M. Cost burden of chronic rhinosinusitis: a claims-based study. Otolaryngol Head Neck Surg. 2011;144(3):440-5.

6. D. Klonaris, M. Doulaptsi, Karatzanis, et al. Assessing quality of life and burden of disease in chronic rhinosinusitis: a review. Rhinology Online. 2018;2(6 - 13).

7. Wright ED, Agrawal S. Impact of perioperative systemic steroids on surgical outcomes in patients with chronic rhinosinusitis with polyposis: evaluation with the novel Perioperative Sinus Endoscopy (POSE) scoring system. Laryngoscope. 2007;117(11 Pt 2 Suppl 115):1-28.
8. Kern RC, Conley DB, Walsh W, Chandra R, Kato A, Tripathi-Peters A, et al. Perspectives on the etiology of chronic rhinosinusitis: an immune barrier hypothesis. Am J Rhinol. 2008;22(6):549-59.

9. Belvisi MG. Regulation of inflammatory cell function by corticosteroids. Proc Am Thorac Soc. 2004;1 (3):207-14

10. Khan A, Vandeplas G, Huynh TMT, Joish VN Mannent L, Tomassen P, et al. The Global Allergy and Asthma European Network (GALEN rhinosinusitis cohort: a large European cross-sectional study of chronic rhinosinusitis patients with and without nasal polyps. Rhinology. 2019;57(1):32-42.

11. Poetker DM, Jakubowski LA, Lal D, Hwang $\mathrm{PH}$, Wright ED, Smith TL. Oral corticosteroids in the management of adult chronic rhinosinusitis with and without nasal polyps: an evidence-based review with recommendations. Int Forum Allergy Rhinol. 2013;3(2):104-20.

12. Shen KH, Wang YH, Hsu TW, Hsieh LC, Sun FJ, Wang YP. Differential effects of postoperative oral corticosteroid on eosinophilic vs. non-eosinophilic CRSwNP subtypes. Am J Otolaryngol. 2019;40(1):22-9.

13. Dautremont JF, Mechor B, Rudmik L. The role of immediate postoperative systemic corticosteroids when utilizing a steroid-eluting spacer following sinus surgery. Otolaryngol Head Neck Surg. 2014;150(4):689-95

14. Rudmik L, Soler ZM, Orlandi RR, Stewart MG, Bhattacharyya N, Kennedy DW, et al. Early postoperative care following endoscopic sinus surgery: an evidence-based review with recommendations. Int Forum Allergy Rhinol. 2011;1(6):417-30.

15. Wong EH, Porter JD, Edwards MR, Johnston SL. The role of macrolides in asthma: current evidence and future directions. Lancet Respir Med. 2014;2(8):657-70.

16. Peric A, Vojvodic D, Matkovic-Jozin S. Effect of long-term, low-dose clarithromycin on $T$ helper 2 cytokines, eosinophilic cationic protein and the 'regulated on activation, normal T cell expressed and secreted' chemokine in the nasal secretions of patients with nasal polyposis. J Laryngol Otol. 2012;126(5):495-502.

17. Huang Z, Zhou B. Clarithromycin for the treatment of adult chronic rhinosinusitis: a systematic review and meta-analysis. Int Forum Allergy Rhinol. 2019;9(5):545-55.

18. Lasso A, Masoudian P, Quinn JG, Cowan J, Labajian V, Bonaparte JP, et al. Long-term low-dose macrolides for chronic rhinosinusitis in adults - a systematic review of the literature. Clin Otolaryngol. 2017;42(3):637-50.

19. Seresirikachorn K, Suwanparin N, Srisunthornphanich C, Chitsuthipakorn W, Kanjanawasee D, Snidvongs K. Factors of success of low-dose macrolides in chronic sinusitis: Systematic review and meta-analysis. Laryngoscope. 2019;129(7):1510-1519.

20. Head K, Chong LY, Piromchai P, Hopkins C, Philpott C, Schilder AG, et al. Systemic and topical antibiotics for chronic rhinosinusitis. Cochrane Database Syst Rev. 2016:4:CD011994.

21. Piromchai P, Thanaviratananich $S$, Laopaiboon M. Systemic antibiotics for chronic rhinosinusitis without nasal pol- 
yps in adults. Cochrane Database Syst Rev. 2011(5):CD008233.

22. Deng J, Chen F, Lai Y, Luo Q, Xu R, Ou C, et al. Lack of additional effects of long-term low-dose clarithromycin combined treatment compared with topical steroids alone for chronic rhinosinusitis in China: a randomized, controlled trial. Int Forum Allergy Rhinol. 2018;8(1):8-14

23. Shen S, Lou H, Wang C, Zhang L. Macrolide antibiotics in the treatment of chronic rhinosinusitis: evidence from a meta-analysis. J Thorac Dis. 2018;10(10):5913-23.

24. Amali A, Saedi B, Rahavi-Ezabadi S, Ghazavi $\mathrm{H}$, Hassanpoor N. Long-term postoperative azithromycin in patients with chronic rhinosinusitis: A randomized clinical trial. Am Rhinol Allergy. 2015;29(6):421-4.

25. Haxel BR, Clemens M, Karaiskaki N, Dippold U, Kettern L, Mann WJ. Controlled trial for long-term low-dose erythromycin after sinus surgery for chronic rhinosinusitis. Laryngoscope. 2015;125(5):1048-55.

26. Varvyanskaya A, Lopatin A. Efficacy of longterm low-dose macrolide therapy in preventing early recurrence of nasal polyps after endoscopic sinus surgery. Int Forum Allergy Rhinol. 2014;4(7):533-41.

27. Zeng $M$, Wang $H$, Liao $B$, Wang $H$, Long $X B_{\text {, }}$ $M a$ J, et al. Comparison of efficacy of fluticasone propionate versus clarithromycin for postoperative treatment of different phenotypic chronic rhinosinusitis: a randomized controlled trial. Rhinology. 2019;57(2):101-9.

28. Albert RK, Connett J, Bailey WC, Casaburi R, Cooper JA, Jr., Criner GJ, et al. Azithromycin for prevention of exacerbations of COPD. N Engl J Med. 2011;365(8):689-98.

29. Han MK, Tayob N, Murray S, Dransfield MT Washko G, Scanlon PD, et al. Predictors of chronic obstructive pulmonary disease exacerbation reduction in response to daily azithromycin therapy. Am J Respir Crit Care Med. 2014:189(12):1503-8

30. Kondo M, Tamaoki J. Therapeutic approaches of asthma and COPD overlap. Allergol Int. 2018;67(2):187-90.

31. Kostadima E, Tsiodras S, Alexopoulos El, Kaditis AG, Mavrou I, Georgatou N, et al. Clarithromycin reduces the severity of bronchial hyperresponsiveness in patients with asthma. Eur Respir J. 2004;23(5):714-7.

32. Simpson JL, Powell H, Boyle MJ, Scott RJ, Gibson PG. Clarithromycin targets neutrophilic airway inflammation in refrac- tory asthma. Am J Respir Crit Care Med. 2008;177(2):148-55.

33. Singh D, Agusti A, Anzueto A, Barnes PJ, Bourbeau J, Celli BR, et al. Global Strategy for the Diagnosis, Management, and Prevention of Chronic Obstructive Lung Disease: the GOLD science committee report 2019. Eur Respir J. 2019;53(5).

34. Maselli DJ, Hardin M, Christenson SA, Hanania NA, Hersh CP, Adams SG, et al. Clinical Approach to the Therapy of Asthma-COPD Overlap. Chest. 2019;155(1):168-77.

35. Oakley GM, Christensen JM, Sacks R, Earls P, Harvey RJ. Characteristics of macrolide responders in persistent post-surgical rhinosinusitis. Rhinology. 2018;56(2):111-7.

36. Wallwork B, Coman W, Mackay-Sim A, Greiff L, Cervin A. A double-blind, randomized, placebo-controlled trial of macrolide in the treatment of chronic rhinosinusitis. Laryngoscope. 2006;116(2):189-93.

37. Tajudeen BA, Ganti A, Kuhar HN, Mahdavinia M, Heilingoetter A, Gattuso P, et al. The presence of eosinophil aggregates correlates with increased postoperative prednisone requirement. Laryngoscope. 2019;129(4):794-9.

38. Kennedy DW. Functional endoscopic sinus surgery. Technique. Arch Otolaryngol. 1985;111(10):643-9.

39. Stammberger $H$. Endoscopic surgery for mycotic and chronic recurring sinusitis. Ann Otol Rhinol Laryngol Suppl. 1985;119:1-11.

40. Soler ZM, Sauer D, Mace J, Smith TL. Impact of mucosal eosinophilia and nasal polyposis on quality-of-life outcomes after sinus surgery. Otolaryngol Head Neck Surg. 2010;142(1):64-71.

41. Brescia G, Pedruzzi B, Barion U, Cinetto F Giacomelli L, Martini A, et al. Are neutrophil-, eosinophil-, and basophil-to-lymphocyte ratios useful markers for pinpointing patients at higher risk of recurrent sinonasal polyps? Am J Otolaryngol. 2016;37(4):33945.

42. Soy FK, Pinar E, Imre A, Calli C, Calli A, Oncel S. Histopathologic parameters in chronic rhinosinusitis with nasal polyposis: impact on quality of life outcomes. Int Forum Allergy Rhinol. 2013;3(10):828-33.

43. Lou H, Zhang N, Bachert $C$, Zhang L. Highlights of eosinophilic chronic rhinosinusitis with nasal polyps in definition, prognosis, and advancement. Int Forum Allergy
Rhinol. 2018;8(11):1218-25.

44. Hopkins C, Gillett S, Slack R, Lund VJ, Browne JP. Psychometric validity of the 22-item Sinonasal Outcome Test. Clin Otolaryngol. 2009;34(5):447-54.

45. Lu W, Qi F, Gao ZQ, Feng GD, Yuan XD, Jin XF. [Quality of life survey on patients with chronic rhinosinusitis by using Chinese version of the 22-item sinonasal outcome test (SNOT-22)]. Zhonghua Er Bi Yan Hou Tou Jing Wai Ke Za Zhi. 2008;43(1):18-21.

46. Lund VJ, Kennedy DW. Staging for rhinosinusitis. Otolaryngol Head Neck Surg. 1997;117(3 Pt 2):S35-40.

47. DeConde AS, Mace JC, Levy JM, Rudmik L, Alt JA, Smith TL. Prevalence of polyp recurrence after endoscopic sinus surgery for chronic rhinosinusitis with nasal polyposis. Laryngoscope. 2017;127(3):550-5.

48. Rowe-Jones JM, Medcalf M, Durham SR, Richards DH, Mackay IS. Functional endoscopic sinus surgery: 5 year follow up and results of a prospective, randomised, stratified, double-blind, placebo controlled study of postoperative fluticasone propionate aqueous nasal spray. Rhinology. 2005:43(1):2-10.

49. Snidvongs K, Lam M, Sacks R, Earls P, Kalish L, Phillips PS, et al. Structured histopathology profiling of chronic rhinosinusitis in routine practice. Int Forum Allergy Rhinol. 2012;2(5):376-85.

50. Sreeparvathi A, Kalyanikuttyamma LK, Kumar M, Sreekumar N, Veerasigamani N. Significance of Blood Eosinophil Count in Patients with Chronic Rhinosinusitis with Nasal Polyposis. J Clin Diagn Res. 2017;11(2):MC08-MC11.

\section{Wang Ying-Piao \\ Department of Otolaryngology \\ Head and Neck Surgery \\ Mackay Memorial Hospital \\ Taipei \\ Taiwan}

Tel: +886-2-2543-3535

Fax: +886-2-2543-3642

E-mail: carlsonwang@yahoo.com.tw 